\title{
An Evaluation of Factors Involved in Perception of Smile Attractiveness of Static and Dynamic Smile
}

\section{Dhiraj Reddy', Dr Ajit Kalia 1, Rajaganesh Gautam ${ }^{1}$ and Lubna Sirkhot ${ }^{1 *}$}

${ }^{1}$ Department of orthodontics, MA rangoonwala dental college, pune

*Corresponding Author Lubna Sirkhot, Department of orthodontics, MA Rangoonwala dental college, pune, India.

Received date: March 25, 2019;Accepted date : April 18, 2019; Published date: May $01,2019$.

Citation: Lubna Sirkhot, An Evaluation of Factors Involved in Perception of Smile Attractiveness of Static and Dynamic Smile, J Dentistry and Oral Maxillofacial Surgery, Doi: http://dx.doi.org/ 10.31579/ jdos.2019/007.

Copyright : (c) 2019 Lubna Sirkhot. This is an open-access article distributed under the terms of The Creative Commons Attribution License, which permits unrestricted use, distribution, and reproduction in any medium, provided the original author and source are credited.

\section{Abstract:}

There Is no consensus on the smile esthetics criteria as yet in published literature. Our study tries to quantify the smile esthetics and test the reliability and repeatability of these criteria. Most studies in smile esthetics have ignored facial movements during smiling. The inferences are derived from still images of the individual's lips and dentition. The esthetics during smiling however, comprises of more dynamic movements, which cannot be represented in a still photograph, where a complete smile cannot be rendered.

Self-image and attractiveness are a person's own perception of their facial appearance and any associated deformity is of great importance

\section{Materials and Methods:}

Eight adult females from Indian population were chosen by an orthodontist from those presenting at our dental college. We finally chose four individuals out of the 8 models that met the established inclusion and exclusion criteria.

The four models were asked to smile in 8 different patterns after providing appropriate training. The variations in smiles were controlled for by careful construction using the facial action coding system. Unwanted variations were reduced using visual monitoring of the activity in action units. The images and videos were evaluated on the perceived smile attractiveness by two rater panels ( 50 dental students and 50 lay persons) who were recruited from our institute.

\section{Results:}

For all raters, Type 4 smile was rated best, followed by the 3,1 and 2 for both the models across all eye related factors ( eye use/non use, eye open/blocked) there was no significant variation in female and male raters esthetic scores Conclusion:

Increased recruitment of muscles in smile production led to increased smile esthetics. The actions of other facial muscles was influenced by eye use.

Keywords: smile evaluation, attractiveness, dynamic, static smile.

\section{Introduction}

According to Jackson, the three main objectives of orthodontic treatment are Functional efficiency, Structural balance and aesthetic harmony which is called as Jackson's triad. These prime objectives in orthodontic treatment are directed towards improvement in function, improvement in aesthetics and the maintenance of these improvements. ${ }^{1}$

It was In the mid-18th century the term "aesthetica" was coined by German scholar Alexander Baumgarten, from the Greek word meaning things perceptible to sense', and soon afterward the concept and the word were applied broadly to the arts and nature. ${ }^{2}$ In the $19^{\text {th }}$ Century, Norman Kingsley, the leading orthodontist of that era, emphasized the aesthetic objectives of orthodontic treatment.

The smiling process involves a considerable part of the facial musculature. Facial muscular activity involved in facial expression can be described by a standard method known as the facial action coding system. This system details 46 unique facial movements known as action units. Gracely et al, differentiated between sensory and affective pain components by developing a box scale. This scale has shown strong correlations across groups, sessions and experiments. It has strong external and internal validity and thus was modified for esthetic ratings for the purpose of our study.
There are two different type of smiles that are evaluated that are dynamic and statics smiles. Static smiles convey structural facial information, while the dynamic smile can convey complex emotions.

Smile attractiveness is a parameter most lay people use to judge the success of the treatment.

Our study aimed to evaluate the factors involved in the perception of smile attractiveness of the static and dynamic smiles.

AIM:To evaluate factors involved in perceived smile attractiveness of static and dynamic smiles.

\section{Materials and Methods}

Subjects to be screened were young adult females from Indian population. The study was approved by the Ethical committee of our institute. Eight female models from Indian population were selected by an orthodontic expert from our dental college and research centre, out of which the final 4 models were selected who met the inclusion and exclusion criteria described below. The 4 models were trained to produce 8 different smiles (Figure 1). 
The following candidates were excluded from the study:

No AU 6

With AU 6
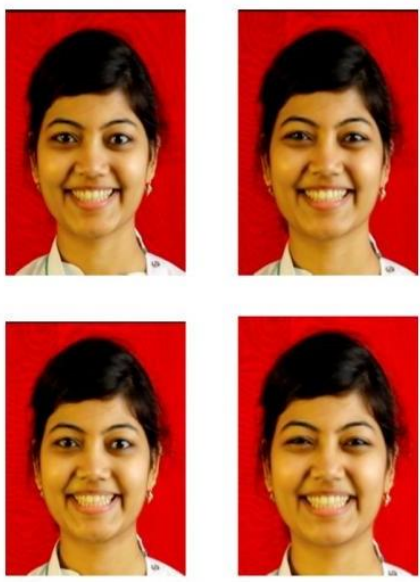

Smile 2

AU 12, 20, 25
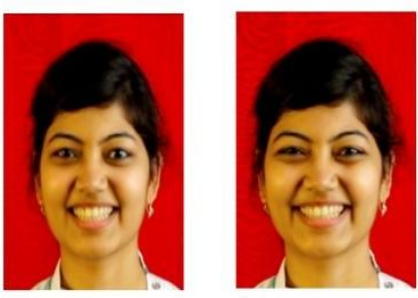

Smile 3

AU 10,12, 25
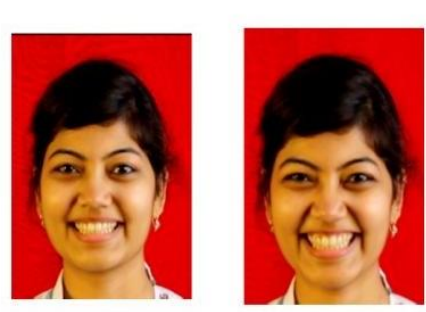

Examples of Smiles 1-4 with and without use of eyes (action unit 6)

Figure:1 Example of 8 different smiles with and without use of eyes (action unit 6).

Two rater panels (50 dental students and 50 lay persons) were recruited from our college. The panel consisted of dental students pursuing internship and lay person consisted of patients who visited our department of orthodontics and dentofacial orthopaedics. The subjects with following criteria were included in the study.

1. Women, between ages of 20 to 30 years;

2. Good facial and skeletal balance based on extra oral examinations;

3. Good general health;

4. Good dental health with functional dentitions.

5. Body mass index within $15 \%$ of the ideal for their age.

6. The models had no history of organic disease, congenital disease, orofacial pain, dysfunction, arthritis, joint noises or restrictions.

7. The models were not under any medications known to interfere with affective or motor parameters.

8. The models were chosen who could follow basic instructions and perform specific different smile patterns after training.

9. To control for sex effects in the interpretation of the results, only female models were chosen for this study.
1. Maxillary incisor angulation $\geq 2 \mathrm{~mm}$ from ideal,

2. Maxillary midline $\geq 4 \mathrm{~mm}$ from the facial midline,

3. Open gingival embrasure between the maxillary central incisors $\geq 2$ $\mathrm{mm}$ (space between the tip of the interdental papilla and the interproximal contact point)

4. Maxillary incisal plane deviation $\geq 1 \mathrm{~mm}$, and

5. Gingival-to-lip distance $\geq 2 \mathrm{~mm}$ at the maximum smile.

\section{Methodology}

\section{STEP-1}

\section{Production of Static and Dynamic smile:}

The study smiles were carefully constructed using the facial action coding system to control for variations in smiles. The activity in action units was monitored visually to reduce unwanted variations in static and dynamic smile.

\section{The action units used included}

1. Action unit 6, -cheek raise, ll contraction of the orbicularis oculi;

2. Action unit 10, -upper lip raiser,\| contraction of the zygomaticus minor and levator labii superioris;

3. Action unit 12, -lip corner puller,\| contraction of the zygomaticus major;

4. Action unit 20, -lip stretch, $\|$ contraction of the risorius; and

5. Action unit 25, -lips part,\|

Parting of the lips was detailed by the facial acting coding system, thus displaying the dentition. The various smiles were checked for absence of nostril flare, forehead and chin muscle movements, ocular movements, glabellar movements, spontaneous blinking, and unwanted lip muscle movement, involving the lips.

One investigator trained the models to produce 8 different types smiles (Figure -1 ) naturally by using different action units combinations .The 8 different types of smile produced are as follows:

1. Smile 1- shows smile produced by model 1, using action unit 12,25 and there was no use of eye component i.e. action unit 6 .

2. Smile 2.-shows smile produced by model 1 , using action unit 12 , 20,25 and there was no use of eye component that is action unit 6 .

3. Smile 3 -shows smile produced by model 1 , using action unit 10 , 12 , and 25 there was no use of eye component that is action unit 6.

4. Smile 4 shows smile produced by model 1 , using action unit $10,12,20,25$ and there was no use of eye component that is action unit 6

5. Smile 5 shows smile produced by model 1 , using action unit 12,25 and there was use of eye component that is action unit 6

6. Smile 6 shows smile produced by model 1 , using action unit 12 , 20 , and 25 and there was use of eye component that is action unit 6

7. Smile 7 shows smile produced by model 1 , using action unit 10 , 12 , and 25 and there was use of eye component that is action unit 6.

8. Smile 8 shows smile produced by model 1 , using action unit $10,12,20,25$ and there was use of eye component that is action unit 6

The models were provided adequate practice required to reproduce the needed movements, spontaneity and timing. All photographs and videos were shot in good lighting revealing the facial contours, by the same photographer in the photography section of the department. Photographs were taken using a Canon EOS 600 DSLR Camera (Figure 2), 18Megapixels, image sensor 22.3X14.9mm, APS-C, CMOS type with maximum output resolution of 5184X3456, having 18$55 \mathrm{~mm}$ macro lens with image stabilizer, 25 meters oblique 8 feet, light sensitivity of 6400ISO and videos were taken using a Panasonic HCX1000 Video Camcorder (Figure 3), 18.47 megapixels, image sensor 1/2.3" MOS Sensor with maximum output resolution of 1920 x 1080 , having 20x Zoom with 4-Drive Leica Dicomar Lens System with image stabilizer- C4K/4K: POWER O.I.S. 


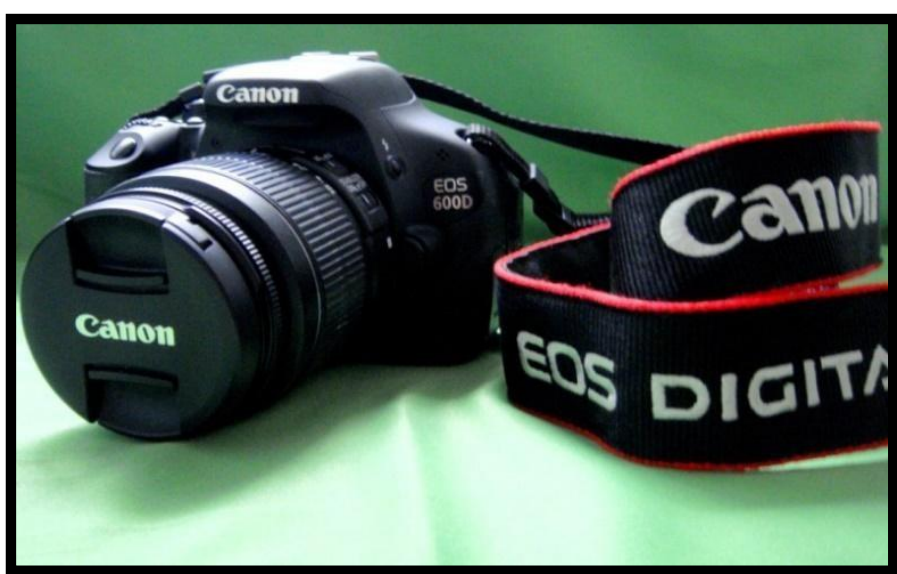

Figure:2: Canon EOS 600 DSLR Camera, 18Megapixels, image sensor $22.3 \mathrm{X} 14.9 \mathrm{~mm}, 18-55 \mathrm{~mm}$ macro lens with image stabilize.

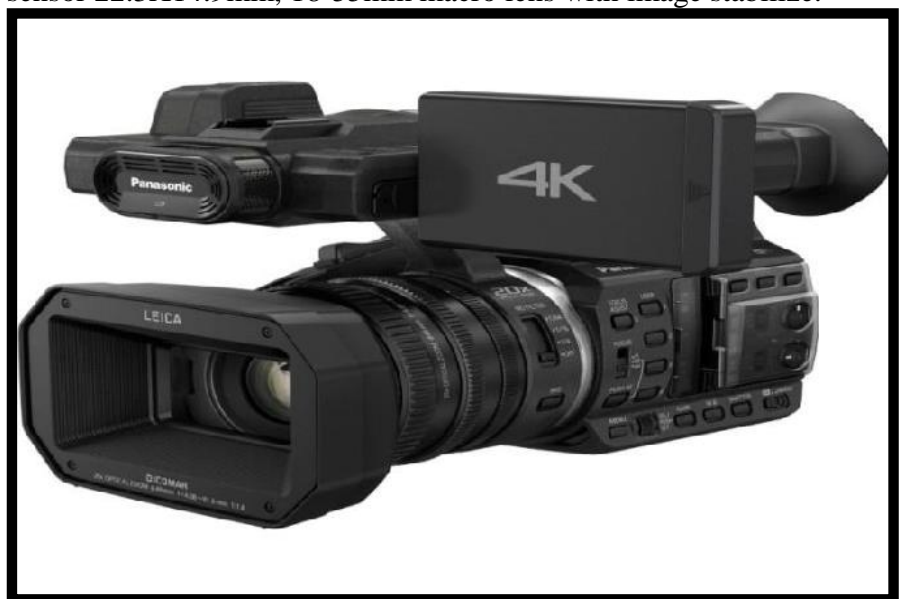

Figure: 3 Panasonic HC-X1000 Video Camcorder, 18.47 megapixels, image sensor 1/2.3" MOS Sensor with maximum resolution of $1920 \mathrm{x}$ 1080, having 20x Zoom with 4-Drive Leica Dicomar Lens System with image stabilizer.

The camera and camcorder were tripod mounted (SIMPLEX $333,175 \mathrm{~cm}, 2.4 \mathrm{~kg}) 36$ inches away from the subject. The patient was asked to be in a Natural Head Position, with eyes looking straight at a distant point. The Natural Head Position (NHP) was recorded with the help of a fluid level device. According to the authors, the sella-nasion to vertical angulation, which is reflected by NHP, can be reliably determined and recorded using this fluid level device. The device was fixed on the right arm of a plastic spectacle such that it occupied the area behind the temporal crest of the frontal bone (Figure 4). The tripod also had a fluid level device attached at the head to orient the camera/camcorder in a balanced plane (Figure 5).

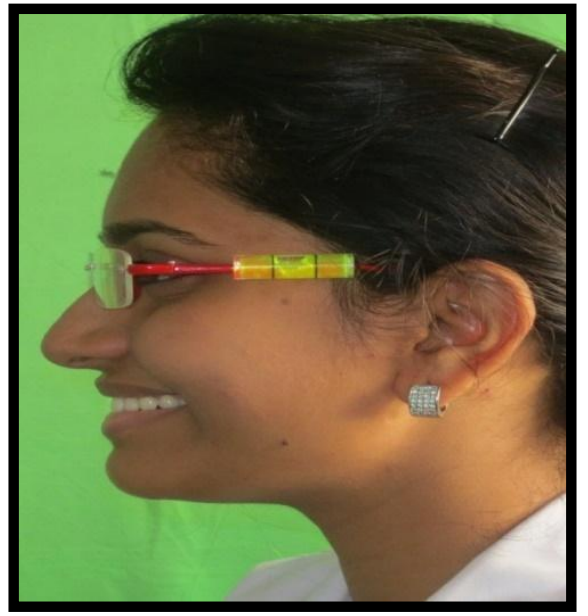

Figure:4 Image and video capture In NHP_with the fluid level device attached to spectacle.

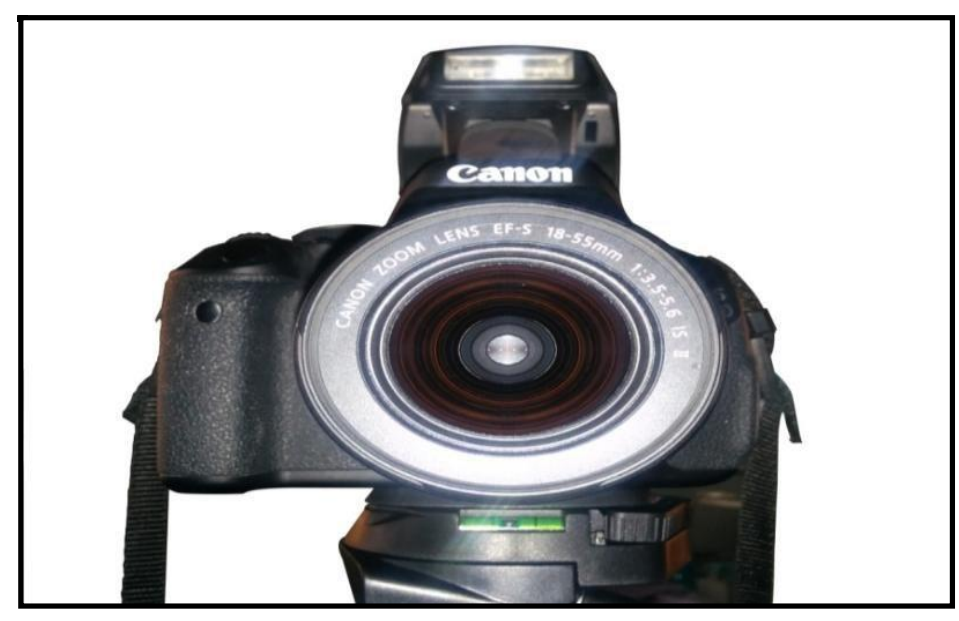

Figure 5: Camera mounted on the tripod. Fluid level device attached to the head of the tripod

Light or bright background, without shadows or distractions were used (Figure 6) before taking the smiling still image/video. A distance of 36 inches was constantly kept between the lens and the subject (Figure 7).

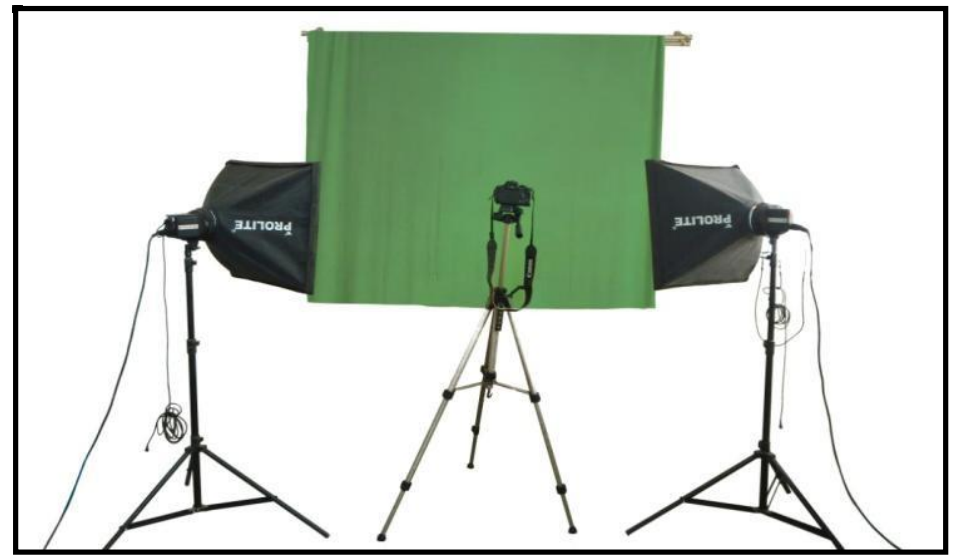

Figure 6: Photographic and video-graphic set up

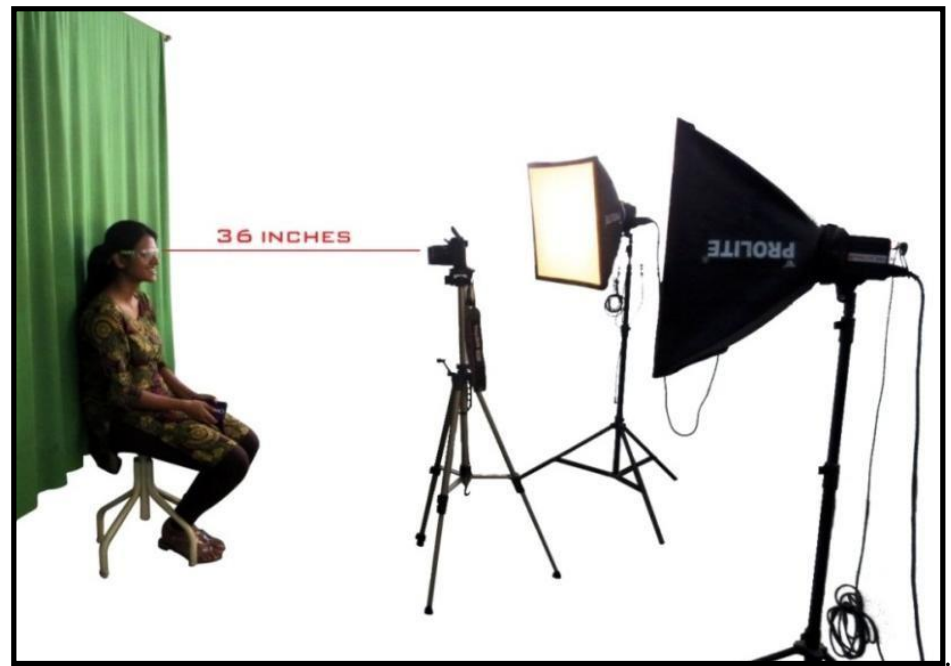

Figure 7: Image and video capture (Distance).

The models were commanded to produce each smile that is to be held at the maximum position for 3 seconds, about the same duration as many human motor events and their perception in humans. Training process lasted between 15 to 30 minutes for every model and it involved having to observe themselves into a mirror, performing designated smile patterns on request. 2 or 3 trials were necessary to record a video clip as well as photographs demonstrating a satisfactory smile. Screening of the models and photographs by the same individual to select the best clip representing each smile for the particular model. 


\section{STEP-2}

\section{Editing of photographs and video clip:}

1. Photographs of 4 models were edited in Adobe Photoshop CS6 Software2014 (Figure 8) and the pictures were edited such that only models face performing smile 4 in the photos were recorded.

2. Videos clips were edited in Key-note (I-work 09-Apple Inc., Cupertino, California, USA) (Figure 9) and converted into 3second individual clips in mov format. Each clip showed the entire head, neck \& shoulder region of the model. The clips were duplicated, and edited with a rectangular block that covering the model's eyes wherever required. This method resulted in 16 videos 8 each for with and without rectangular blocks for each model.

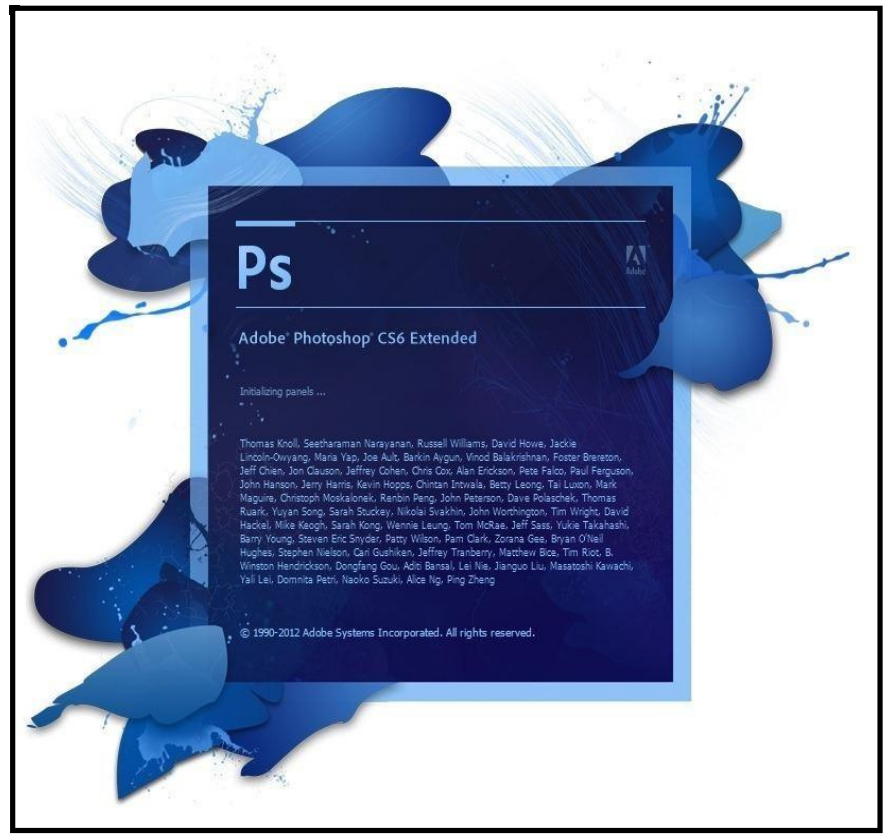

Figure 8: Adobe Photoshop CS6 Software2014 (Microsoft Corporation San Jose, California, USA).

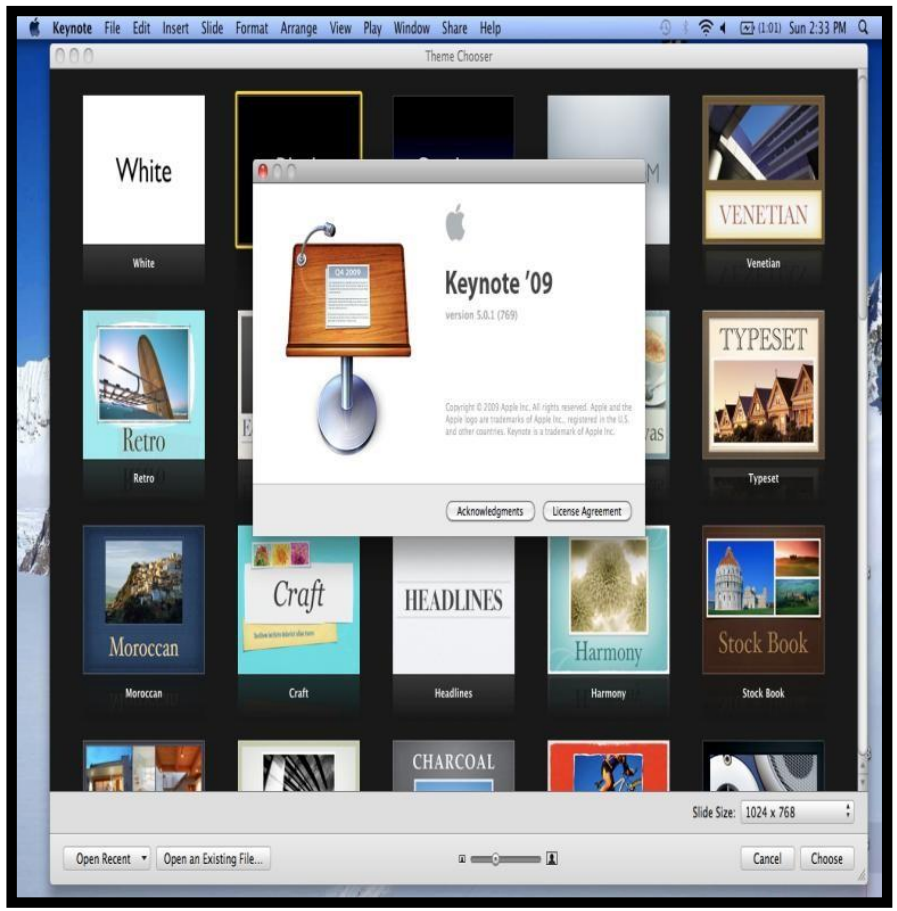

Figure:9 Key-note ( I-work 09-Apple Inc, Cupertino, California, USA).

Auctores Publishing - Volume1-007 www.auctoresonline.org Page - 04

\section{STEP- 3}

The computer based survey consist of 5 parts as follows.

Part 1 consisted of Participating Rater's demographic data( Figure 16). The rater's demographic information includes sex, age, ethnicity and occupation were collected.

Part 2 consist of the preliminary pilot study. In this part, the models overall facial attractiveness, photographs of the 4 models were developed on computer screen (Figure -10). The pictures displayed only the models heads, and all models performing smile 4 in the images. The participants were asked to rate each model's overall facial attractiveness on rating sheet (Figure 17) with a VAS (VISUAL ANALOGUE SCALE) (Figure 11) scale of 0 to 10 .with zero indicating the least attractive and ten indicating the most attractive. Gracely et al ${ }^{32,33}$ developed a box scale for differentiating between the sensory and affective pain components. Because of its power and validity as a rating method, the scale was modified to provide aesthetic ratings in our study. Based on these ratings in the preliminary pilot study, ratings of attractiveness of models 1 and 3 were similar to one another, while the ratings of models 2 and 4 were similar to each other. As a consequence, only one of models 1 and 3 and only one of models 2 and 4 that is model 1 and 2 were randomly assigned to every rater for the third and fourth part of the study.

Part 3 consisted of 32 videos ( 16 per model of 2 models) being presented to each rater thrice in random order to control for spatial order effects, acquiring 96 overall responses. The raters chose the number in the VISUAL ANALOGUE SCALE (VAS) ${ }^{32,33}$ (Figure 12) corresponding to their rating of the attractiveness of each smile in manual rating sheet (Figure 18).

Part 4 consisted of forced comparison, with 2 videos being presented simultaneously, where the raters had to choose between the 2 smiles based on the attractiveness (Figure 19). The paired videos were selected randomly according to certain rules:

(1) The pair represented the same model.

(2) The pair differed from the other in only one of 3 ways-

A. Type of smile (smile 1, 2, 3, or 4)

B. Use of the eyes (action unit 6 used or not used)

C. Blocking of the eyes (eyes blocked or eyes visible).

This method gave 40 paired videos representations for each model: i.e, 2 eye-use conditions times, 2 eye-blocking conditions times 10 smile pairs - smiles 1 and 1,1 and 2,1 and 3,1 and 4,2 and 2,2 and 3,2 and 4, 3 and 3,3 and 4 , and 4 and 4 .

To control for spatial order effects, each of the 40 video pairs were displayed twice in random order on the screen. Thus the raters viewed a total of $80(40 \times 2)$ pairs of smile videos for every model. Overall 160 total presentations were rated as each rater viewed the smiles of 2 models in the manual rating sheet.

Part 5 consisted of overall facial attractiveness of models. At the end of the survey, the raters were again asked to rate photographs of only smile four of all four models (Figure 14), each model's overall facial attractiveness on a scale of 0 to 10 in manual rating sheet (Figure 20). The presentation of this part was identical to part 2 .

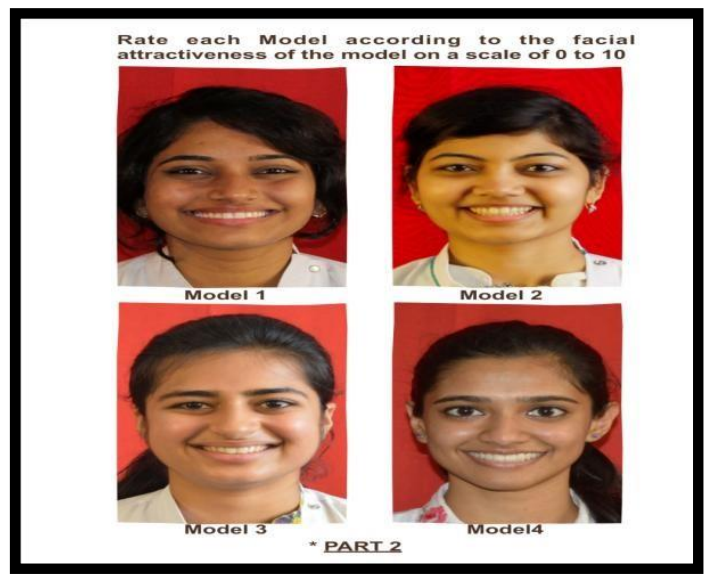

Figure:10 Part 2-Checklist. 

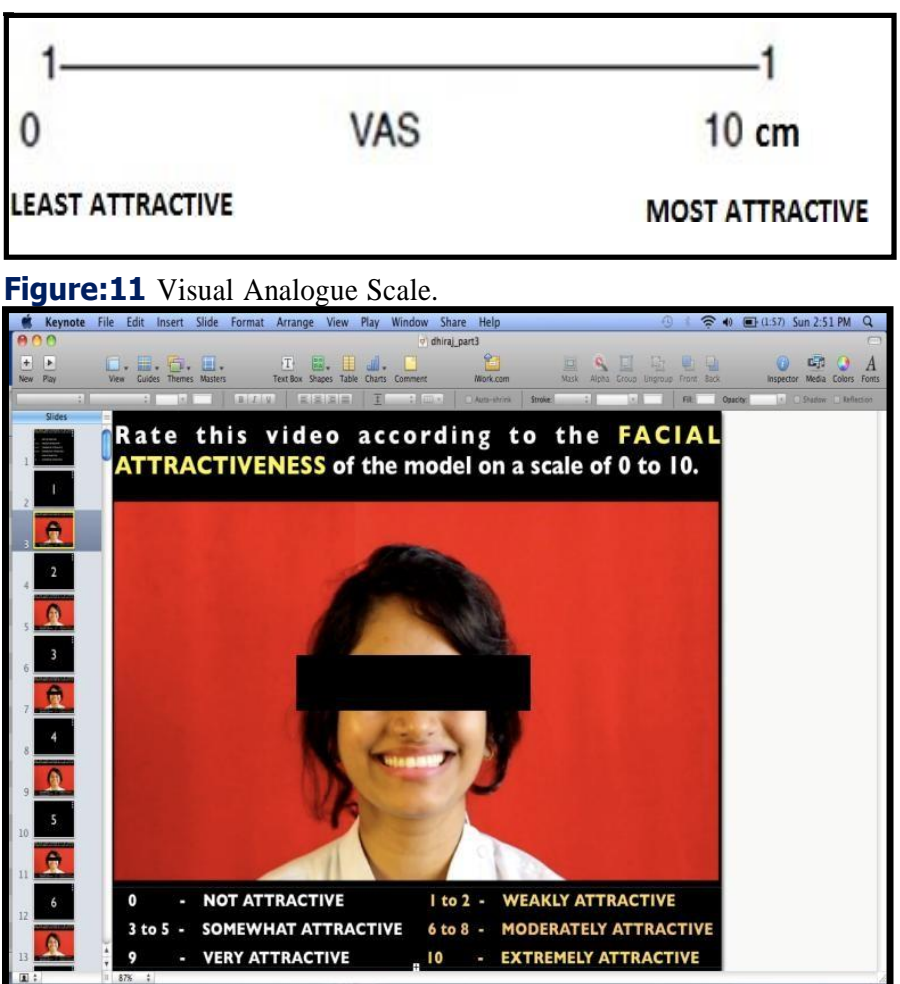

Figure:12 Part3-Checklist.

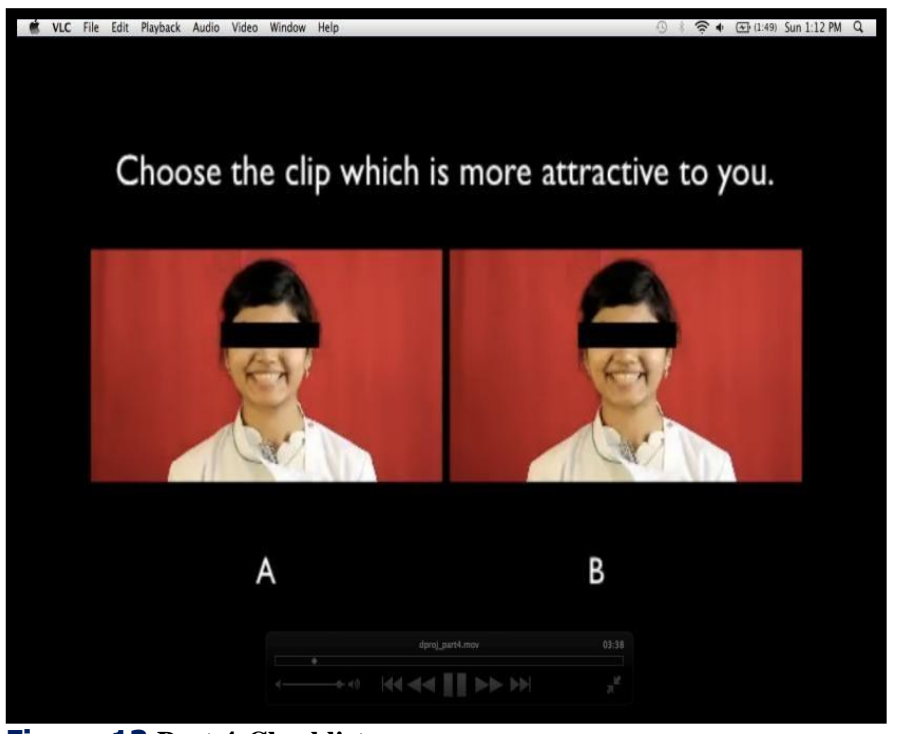

Figure:13 Part 4-Checklist.

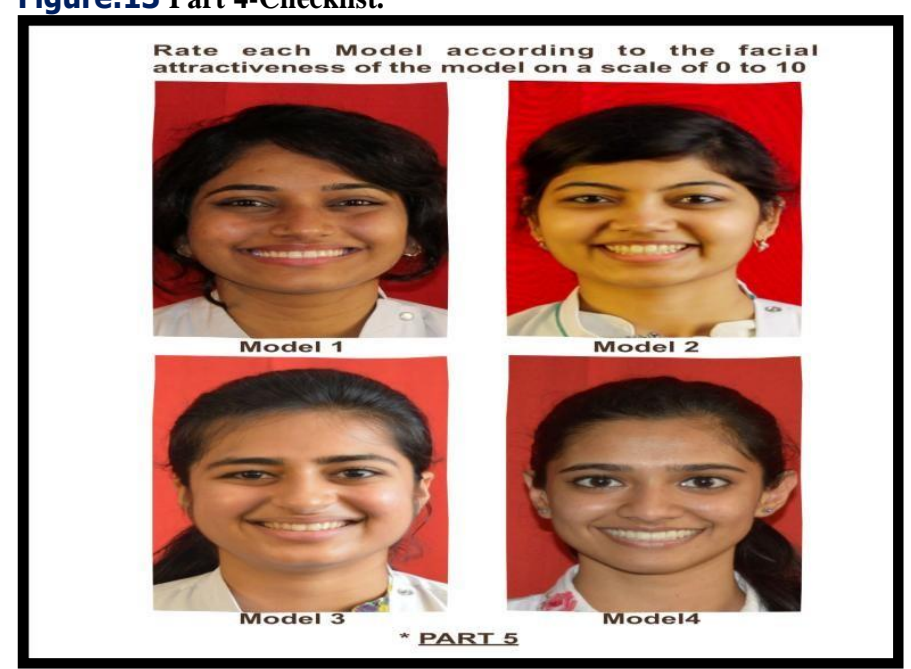

Figure:14 Part 5-Checklist.

\section{STEP-4 \\ Rating of photographs and videos:}

Two rater panels (50 dental students and 50 lay persons) were recruited from our college. The dental panel comprised of dental students pursuing their internship and a lay person comprised of a random patient who presented at our department of orthodontics and dentofacial orthopaedics. Participants presenting above the age of 18 years were invited to participate.

A total of 100 judges (i.e. 50 dental student and 50 Lay persons) were given a C.D. (COMPACT DISC, SONY-700mb)(Figure-15) each. The C.D. contained the following data:

1. Part-2 (4 Photographs of 4 different models).

2. Part-3 (A combined clip of 96 videos).

3. Part-4 (A combined clip of 160 videos, each comparing two videos in random order for assessment purpose).

4. Part-5 (4 Photographs of 4 different models).

Each rater was then asked to view the $\mathrm{CD}$ on a computer and instructions for assessing each part of study were given in the beginning of each of the clip in the $\mathrm{CD}$. The raters were asked to mark his or her assessments of the facial attractiveness of the subject on the $10 \mathrm{~mm}$ visual analogue scales, which were anchored by the descriptors -Least Attractivell (0) and -Most Attractivell (10). Visual analogue scales ${ }^{32,33}$ have been found to provide rapid, convenient, valid, reproducible, and representative ratings of dental and facial appearance. The raters were given specific instructions on the use of the scale. This evaluation sheet contained Part-1 checklist (demographic data of the raters) (4), Part- 2 checklist, Part- 3 checklist, Part- 4 checklist and Part- 5 checklist.

\section{STEP-5}

\section{Statistical Analysis:}

Statistics analysis were calculated for the raters including sex, age and ethnicity. Non-Parametric tests were used to analyses the VAS -scale data (part 2,3,4 and 5).

Part 3: The median score for each smile was plotted to identify rating trends that might have existed across the 3 presentations of each video. All data were chosen for analysis using Mann-Whitney test (Minitab version 17.2) to study effects from smile type, use of action unit 6, blocking of eyes, rater panel, rater age, and rater sex.

Data from the forced comparison (part 4) were analyzed by using one proportion test (Minitab version 17.2) (is proportion> 0.5?). For this study,3 forced-comparison analyses were performed using the one proportion test (Minitab version 17.2) (proportion>0.5).

1. Comparing the 4 smile types while holding -use of action unit $6 \|$ and -blocking of eyes\| constant;

2. Comparing -use of action unit $6 \|$ while holding smile type and -blocking of eyes\| constant;

3. Comparing -blocking of eyes\| while holding smile type and -use of action unit $6 \|$ constant.

As an example, to compare smile types while holding -use of action unit $6 \|$ and -blocking of eyes $\|$ constant, we first determined for each rater the number of times each smile type was selected in the forced comparison with every combination of eye use and eye block.

\section{Results:}

In our study, eight adult females from Indian population were selected by an orthodontic expert from our dental college, out of which the final four models were selected who met the inclusion and exclusion criteria.

The four models were trained to produce 8 different smiles (Figure 1). The study smiles were carefully constructed using the facial action coding system to control for variations in smiles. The activity in action units was monitored visually to reduce unwanted variations in static and dynamic smiles. The images and videos were evaluated on the perception of smile attractiveness by two rater panels (50 dental students and 50 lay persons) who were recruited from our college. The dental panel consisted of dental students pursuing internship and lay panel consisted of adult patients who visited our department of orthodontics. 
For part 1, rater participant data, 100 raters took the survey: 50 in the dental panel and 50 in the lay panel .The age range (21-26 years) were young adults in both the panels; therefore, age was not deemed a relevant factor for investigation in our study. Other descriptive statistics about the raters is as shown in Table -1 .

In part 2, overall facial attractiveness of the models, photographs of the 4 models were presented on the same page on the computer screen. The pictures showed only the model's head, and all models were performing smile 4 in the photograph. The participants were asked to rate each model's overall facial attractiveness on a VAS scale of 0 to 10 , with score 0 being the least attractive and score 10 being the most attractive.

The results of part 3 and part 4 of study are presented as bar graphs in figures. In these graphs the smiles produced by the two models with two eye use conditions ( with and without use of AU 6) and two eye block conditions (with and without eye block) are numbered as follows and which are plotted along the $\mathrm{X}$ axis in the graphs.

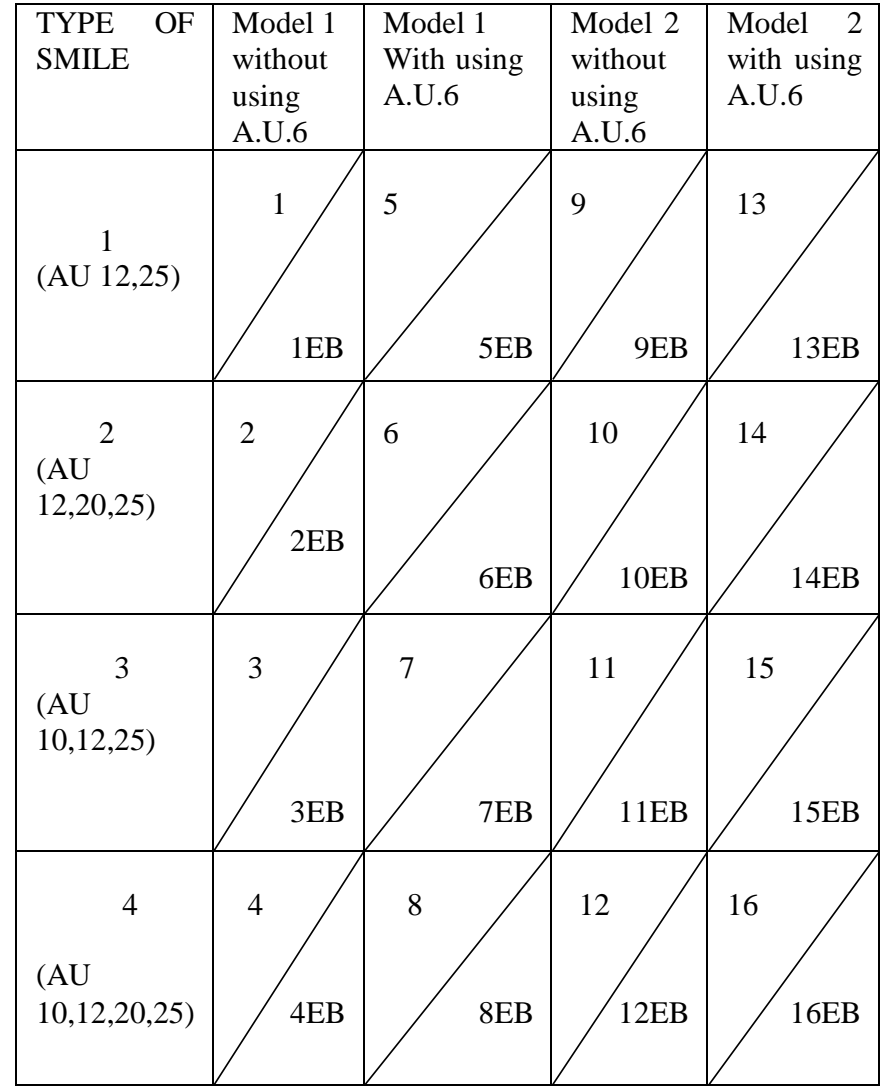

Further for the above 16 smiles with EYE BLOCK condition, a similar labelling with the suffix EB is used. For example Smile $1 \mathrm{~EB}$ : Smile produced by Model 1 using action units 12 and 25, and without eye use (Action unit 6) and with the eyes blocked. Thus similarly Smile 2 EB, Smile 3 EB and so on are labelled till Smile 16 EB. For ease of understanding the above nomenclature, the following table may be referred while reading the results.

In part 3, the bar graphs showed the distribution of VAS scale scores for each of the 4 smiles, pooled across models and eyerelated factors. Therefore, non-parametric statistics were used to analyses the VAS scale data. Each smile was presented 3 times in the screen during study portion of the survey. The scores from the all the presentations were used in analyses. The results for this part of our study are as follows. For all raters, Type 4 smile was rated best ,followed by the 3,1 and 2 for both the models across all eye related factors ( eye use/non use, eye open/blocked)( Figure 22)

The above sequence of rater preference for the smiles did not vary significantly depending on the rater's age, sex and types of raters. (Dental and lay people)(Figure 23, 24 and 25 respectively.)
Among lay and dental raters, contracting the orbicularis oris (using action unit 6) caused an increase in the scores for all the smile types, for both the models in eye open condition, but the effect was statistically insignificant.

For eye blocked condition, contracting the orbicularis oris (using action unit 6) caused a increase in the scores for model 1 (Table 3) and 2 (Table 4)in all types except Type 2 smile in model 2 ,but again the differences were not statistically significant.

In part 4 , results for the forced comparison appear which represent the combined data from all the 100 raters and shows the maximum occurrence i.e median, for selection of a given smile type. Here the results show that type 4 smile was preferred the most followed by 3,1 , and finally type 2 smile, for both the models across all eye related factors (eye use/non use, eye open/blocked) (Figure 26-33).The above results in this part of the study (forced comparison) also did not vary across rater panel (dental/ lay), age and sex.

The forced comparison results for the different smile types in each model, for the various eye use conditions is as described below.

1) Forced comparison in Model 1 between "without using AU 6" versus "with using AU 6"conditions : (Table 5 and 6)

Type 1 smile: Percentage of selecting Type 1 smile decreased by $24.75 \%$ $(46.34-21.59=24.75)$ without eye block and increased by $40.53 \%(25.63$ $66.16=40.53$ ) with eye block condition

Type 2 smile: Percentage of selecting Type 2 smile Increased by $12.63 \%($ $3.03-15.66=12.63)$ without eye block and decreased by $8.33 \%(33.33-$ $25.00=8.33$ ) with eye block condition.

Type 3 smile: Percentage of selecting Type 3 smile increased by $3.91 \%($ $56.94-75.76=3.91)$ without eye block and decreased by $0.38 \%(49.75$ $49.37=0.38$ ) with eye block condition.

Type 4 smile : Percentage of selecting Type 4 smile increased without eye block and with eye block condition by $17.93 \%(75.76-93.69=17.93)$ and $6.06 \%(75.00-81.06=6.06)$ respectively.

In part 5, overall facial attractiveness of models, at the end of the survey, the raters were asked once again to rate overall facial attractiveness on a scale of 0 to 10 of all four models The presentation of this part was identical to part 2.

The results of this part were also similar to that obtained in part 2 namely, that the scores for Models 1 and 3 (Figure 34) were similar and statistically insignificant(able 9) and scores for models 2 and 4(Figure 34) were similar and statistically insignificant (Table 9). However the p values obtained in this part 5(Table 9) were different than those in part 2(Table 2).

The data from this part 5 was then used to test our 4th objective which was whether there are differences in the perceived smile attractiveness of static and dynamic smiles of the same individual. In our study we compared the static and dynamic smiles of Model 1 by comparing results of smile 4 of this model from study part 5 with that from study part 3 respectively. The dynamic smile of this model showed a higher median score than the static smile (Figure 35), by both the rater panels.

\section{Discussion:}

Smile plays a critical role in aesthetics and social behavior. It is an important component in orthodontic treatment management. This has been recognized since the beginning of the specialty and in the current aesthetically oriented society, it seems to play a central part in selfperception and social image. The smile is rightfully considered a valuable tool of nonverbal social communication, a civilized form of human contact, and a sound criterion of facial attractiveness. Historically, orthodontic management largely relied on occlusal relationship results. However, modern orthodontics treatment planning demands a balance between soft tissues and occlusion. Aesthetic preference hierarchy can be identified by studying the various components of perceived smile attractiveness, making it an area of crucial importance in orthodontic management. The last few decades have seen a reemergence of the soft tissue paradigm in orthodontics thus making it an important part of assessment. 
Most of the published literature on smile esthetics has tended to ignore the importance of various facial movements in smiling, with results relying on interpretation of still images showing only the individuals lips and dentition. The still images are an inadequate representation as they do not capture the various dynamic movements that are involved in smiling. The transition from the phases cannot be captured on a still photograph.

Ackerman et al (1998) ${ }^{45}$ devised a method to measure the smile characteristics of patients seeking orthodontic treatment. 'Posed smile' is usually measured as it is voluntary and not emotionally elicited. It can be a learned response of appeasement and is sustainable while also being reliably repeatable. They suggested that the photographic analysis of an unstrained posed smile is considered to be a standard orthodontic record tool. Marc Ackerman and James Ackerman $(2002)^{10}$, in their review of smile analysis and design in the digital era emphasized the key importance of Smile analysis and smile design in orthodontic management in recent years. Recent technological advances allows the clinician to measure dynamic liptooth relationships and utilize the information to devise a problem list and a treatment plan. Digital videography is particularly useful in both smile analysis and in doctor/patient communication. Smile design is a multifactorial process, with clinical successful treatment requiring a complete understanding of the patient's soft tissue limitations and the extent of meeting the patients desired aesthetic goals. The concept of an -ideall smile does not exist. However treatment could help the patient to achieve a more -balancedll smile, which can best be described as an appropriate positioning of the teeth and gingival scaffold within the dynamic display zone. Studies concluded that Smile analysis and smile design often involve a compromise between two factors that often seem contradictory to the aesthetic desires of the patient, orthodontist, and the patient's structural limitations. Using digital video computer technology has enabled the clinician to incorporate the patient's dynamic smile analysis into treatment planning.

Rubenstein AJ. (2005) $)^{25}$ conducted a study on "Variation in perceived attractiveness: differences between dynamic and static faces." and studies have attempted to define facial attractiveness usually so in terms of structural features of the face (e.g., symmetry, averageness). These studies, however usually use still images of faces which may not be analogous to dynamic faces commonly used in other areas of attractiveness research, such as research investigating the impact of attractiveness on social interaction. Current studies have investigated the similarities and differences of perception and evaluation of dynamic and static images. Studies have demonstrated that dynamic and static faces are judged by different evaluative standards. Other studies demonstrated that perceived emotion may be a key factor in judging the attractiveness of dynamic as compared to static faces. These findings demonstrate further exploration of the differences between dynamic and static faces thereby facilitating a deeper understanding of the characteristics that affect perceived attractiveness.

Pieter A. A. M. van der Geld el al (2007) $)^{54}$, concluded that the videographic method was reliable for measurement of tooth display and lip position in spontaneous and posed smiling and speaking. Application of the method is warranted especially when obtaining an emotional smile is difficult, such as cleft lip and palate or disfigured patients.

Smile esthetics are influenced by the subjective preferences of a viewer and by objective measures of a smile. This study looked at objective model factors and subjective rater factors that were involved in evaluation of perceived smile attractiveness of dynamic and static smiles.

The results of this study showed the type of smile, the use of eyes, and the blocking of eyes as model factor which are common across all models and all raters, regardless of the raters' sex, panel. Our study results showed that smile aesthetics increased with increased recruitment of muscles involved in smile production led to improved smile esthetics.
The use of the orbicularis oculi while smiling had a positive effect on all the smiles studied. However, in contrast with a previous study ${ }^{64}$, our study did not show a preferential increase in the scores of the less attractive smiles when compared to the more attractive smiles, with the use of eyes. This finding of the positive eye effect is in concordance with other studies, ${ }^{63}$ where the use of the orbicularis oculi was identified as intensifying the emotional valence. Our study results also showed that the raters could relate this muscle activity to better smile aesthetics, thus underscoring the relationship between smile attractiveness and positive emotional valence. Our method of blocking the eyes involved covering only the eyes. Blocking the eyes had little effect on the above ratings with both the panel of raters giving an increased score with eye usage, even with eyes blocked condition. This suggests that actions of the orbicularis oculi showed through the smile irrespective of whether a rater could directly see the model's eyes. The use of orbicularis oris tended to make each smile larger ${ }^{64}$ and our study methodology could prove that this usage is picked up by the raters as a subtle difference enhancing the smile score in the different models even in a condition when the eyes are blocked during the assessment. The rater factors in this study were raters panel and sex. These factors represent rater dependent preferences or tastes in beauty. In our study rater dependent factors such as preferred lay person or gender did not impact the discussed results. This shows that rating dynamic smiles were based on objective criteria which remained uniform across the rater population in this study.

In a previous study ${ }^{40}$, female raters gave higher esthetic scores than did the men in almost all cases. Two speculations which were made in that study with regard to this sex difference:

(1) Women may tend to be less critical of facial attractiveness of other women than men,

(2) The sex difference observed may reflect the differential sex preference of various facial attributes: i.e., certain models may have had certain facial features that appealed more to the female than to the male participants supporting the study of Meerdink et al ${ }^{40}$ who stated that men and women focus on different features when assessing the attractiveness of faces. Furthermore, Flores-Mir et al ${ }^{51}$ demonstrated that men are less critical than women in rating facial attractiveness when presented with still images of smiles. In our study, there is no significant variation in female and male raters esthetic scores. In an earlier study also by Parekh et al $(2006)^{71}$ showed no significant differences between the perceptions of female and male raters and Springer et al $(2011)^{72}$ also concluded that the rater's sex was not critical in the evaluation of smile aesthetics.

We used the Visual Analogue Scale (VAS) in our study to score the different images. This scale that has been used to assess pain intensity, is a simple, rapid, valid, and reliable method for raters to judge aesthetics or attractiveness. The greatest advantage of the visual analogue scale does not restrict the raters to categorized assessment; hence, it was preferred in this study. Previous studies have pointed to cultural differences in the perceptions of smile aesthetics among various lay populations. ${ }^{62}$ Our study showed no significant difference in the dental and lay person's score. There were no differences in ethnicity of the raters in our study (all Indians) and hence there was no cultural differences in the perception of smile aesthetics among the raters .Previous studies have mentioned the importance of digitally measuring the smile characteristics of orthodontic patients ${ }^{45}$. The perceived emotion may be more salient in judging the attractiveness of dynamic faces than in judging the attractiveness of static faces $^{25}$. Thus using digital video and computer technology, the clinician can evaluate the patient's dynamic smile analysis into routine treatment planning ${ }^{10}$. This suggests the importance of including videography over photography as a diagnostic tool for a successful orthodontic treatment. Our study also states the importance of dynamic facial aesthetics and its measurement and evaluation with the use of digital videos as more reliable and valuable tool over static photographs in achieving an outcome amenable to both the doctor and patient alike. It should be taken into consideration that sample size was small compared to entire Indian population size. In this study, panel of evaluators of dental students and laypersons may not be representative of the entire population. Also study was of female population only and there was variation in ethnic as well as on regional basis in Indian population. 


\section{Limitation and future scope of study:}

There were some limitations in this study that should be recognized. Because we used specifically defined rater groups-lay persons and dental students - its external validity is limited. It should be taken into consideration that sample size was small compared to entire Indian population size; Also study was of only female population. Therefore, future studies should be conducted to further test the external and ecologic validity and psychometrics of these methods in orthodontics. Also, since aesthetics and their interpretations is subjective it can vary depending on demographic factors, future studies with larger sample size and models and raters representing broader demographic attributes will be required to determine how the findings generalize. 51,62

\section{Conclusion:}

The concept of smile aesthetics is not a new one, as the literature review has shown. Clearly, its impact on the final facial and smile appearance can be quite dramatic. This demands that we rethink some of our orthodontic mechanics and concepts of treatment to consistently build this factor into our diagnostic and treatment planning regiments. This study used photographs of static and video clips of dynamic smiles to evaluate factors involved in perceived smile attractiveness and the following conclusions were reached:

1) Smile aesthetics increased with increased recruitment of muscles involved in smile production

2) The ratings were higher when the eyes (orbicularis oris) were used, irrespective of whether the eyes were visible to the raters, suggesting that influence of eye use on the other facial muscles.

3) The above results were robust across the models and rater panels, suggesting that objective methods are employed in rating of smile-dynamic aesthetics, which were common across dental professionals and lay persons and thus could become an important clinical tool.

4) Our study showed that there are differences between perceived smile attractiveness of static and dynamic smile of the same individual.

\section{References:}

1. Richard A. Riedel. Esthetics and Its Relation to Orthodontic Therapy. The Angle Orthodontist.1950; 20( 3):168-178.

2. Peck S. Peck L. Selected aspects of the art and science of facial esthetics. Seminars in Orthodontics. 1995;1:105-126.

3. Angle EH. The treatment of malocclusion of the teeth. 7 th ed. Philadelphia: SS White.1907:1964.

4. Holdaway RA. A soft-tissue cephalometric analysis and its use in orthodontic treatment planning. Part I. American Journal of Orthodontics. 1983;84:91.

5. Sarver DM, Ackerman MB. Orthodontics about face: the reemergence of the esthetic paradigm. American Journal of Orthodontics and Dentofacial Orthopedics. 2000;117:575-576.

6. Ackerman JL, Proffit WR, Sarver DM. The emerging soft tissue paradigm in orthodontic diagnosis and treatment planning. Clinical Orthodontics and Research. 1999;2:49 52.

7. Burstone CJ. Lip posture and its significance in treatment planning. American Journal of Orthodontics. 1967;53:262-284.

8. Profit WR, Fields HW, Sarver DM, editors. Contemporary Orthodontics. $4^{\text {th }}$ Ed. St Louis: Mosby Elsevier; 2007.

9. David M. Sarver. The importance of incisor positioning in the esthetic smile: The smile arc. American Journal of Orthodontics and Dentofacial Orthopedics. 2001;120:98-111.

10. Ackerman MB, Ackerman J. Smile analysis and design in the digital era. Journal of Clinical Orthodontics. 2002;36:221-236.

11. Janzen EK. A balanced smile - a most important treatment objective. American Journal of Orthodontics.1977; 72:359372.

12. Kokich VO Jr, Kiyak HA, Shapiro PA. Comparing the perception of dentists and lay people to altered dental esthetics. Journal of Esthetic Dentistry. 1999;11(6):311-324.
13. Hulsey CM. An esthetic evaluation of lip-teeth relationships present in the smile. American Journal of Orthodontics.1970;57:132-144.

14. Morley, J. and Eubank, J.: Macroesthetic elements of smile design, Journal of the American Dental Association. 2001;132:39-45.

15. Sarver DM, Ackerman MB. Dynamic smile visualization and quantification: Part 1. Evolution of the concept and dynamic records for smile capture. American Journal of Orthodontics and Dentofacial Orthopedics. 2003;124(1):4-12.

16. Sarver DM, Ackerman MB. Dynamic smile visualization and quantification: Part 2. Smile Analysis and Treatment Strategies. American Journal of Orthodontics and Dentofacial Orthopedics. 2003 ;124(2):116-127.

17. Farhad B. Naini. Facial aesthetics: Concepts \& Canons. Dental update. 2008; 35(2):102-4, 106-107.

18. Tarantili VV, Halazonetis DJ, Spyropoulos MN.The spontaneous smile in dynamic motion. Am J Orthod Dentofacial Orthod. 2005;128:8-15.

19. Rubenstein AJ. Variation in perceived attractiveness: differences between dynamic and static faces. Psychol Sci. 2005;16:759-762.

20. Ekman P, Friesen WV.A tool for the analysis of motion picture film or video tape. Am Psychol.1969;24:240-243.

21. Ekman P. Facial expressions of emotion: an old controversy and new findings. Philos Trans R Soc Lond B Biol Sci.1992;335:6369.

22. Steiner F. Differentiating smiles. In: Branninger-Huber E, Steiner F, editors.FACS in psychotherapy research. Zurich, Switzerland: Department of Clinical Psychology, Universitat Zurich; 1986. p. 139-148.

23. Ekman P, Davidson RJ, Friesen WV. The Duchenne smile: emotional expression and brain physiology. II. J Pers Soc Psychol. 1990;58:342-353.

24. Gracely RH, Dubner R, McGrath P, Heft H. New methods of pain measurement and their application to pain control. Int Dent J. 1978;28:52-65.

25. Gracely RH, McGrath F, Dubner R. Ratio scales of sensory and affective verbal pain descriptors. Pain 1978;5:5-18.

26. Weurpel Edmund. On facial balance and Harmony. The Angle Orthodontist. 1937;7:81-89.

27. Peck H, Peck S. A concept of facial esthetics. The Angle Orthodontist. 1970;40:284-299.

28. Dorsey J, Korabik K. Social and psychological motivations for orthodontic treatment. American Journal of Orthodontics. 1977;72: 460-467.

29. Shaw WC. The influence of children's dentofacial appearance on their social attractiveness as judged by peers and lay adults. American Journal of Orthodontics. 1981; 79:399-415.

30. Lisa A. Tedesco, John J. Cunat et al A dental-facial attractiveness scale. American Journal of Orthodontics. 1983; 83: 38-43.

31. Howells DJ, Shaw WC. The validity and reliability of ratings of dental and facial attractiveness for epidemiologic use. American Journal of Orthodontics. 1985;88:94

32. Ronald J. Mackley. An evaluation of smiles before and after orthodontic treatment. The Angle Orthodontist. 1993; 63:183-189.

33. Johnson DK, Smith RJ. Smile esthetics after orthodontic treatment with and without extraction of four first premolars. American Journal of Orthodontics and Dentofacial Orthopedics. 1995; 108:162-167.

34. Peerlings RH, Kuijpers-Jagtman AM, Hoeksma JB. A photographic scale to measure facial aesthetics. European Journal of Orthodontics. 1995;17:101-109.

35. Kokich VG. Esthetics: the ortho-perio-restorative connection. Seminars in Orthodontics. 1996; 2(1):21-30.

36. Ackerman JL, Ackerman MB, Brensinger CM, Landis JR. A morphometric analysis of the posed smile. Clinical Orthodontics and Research. 1998;1:2-11.

37. Dong JK, Jin TH, Cho HW, Oh SC. The esthetics of thesmile: a review of some recent studies. International Journal of Prosthodontics.1999;12(1):9-19. 
38. Langlois J. H., Kalakanis L., Rubenstein A. J., Larson A., Hallam M., \& Smoot M. Maxims or myths of beauty? A metaanalytic and theoretical review. Psychological Bulletin.2000;126, 390-423.
39. Sarver DM. Principles of cosmetic dentistry in orthodontics: Part Shape and proportionality of anterior teeth. American Journal of Orthodontics and Dentofacial Orthopedics. 2004;126(6):749-753. 